\section{Adding aspirin to antipsychotics reduces psychopathology in adults with schizophrenia spectrum disorders}

\section{QUESTION}

Question: Does adjuvant aspirin benefit patients with schizophrenia spectrum disorders who are on antipsychotic therapy?

Patients: 70 adults (18-55 years of age) with DSM-IV schizophrenia spectrum disorder (schizophrenia, schizoactive disorder or schizophreniform disorder), who were at least moderately ill (score $\geq 60$ on the Positive and Negative Syndrome Scale, PANSS, with score $\geq 4$ on two items). There was a 2 -week placebo run-in period, and only those who achieved over $80 \%$ compliance were randomised. Exclusion criteria: illness duration longer than 10 years (changed from 5 years because of slow recruitment), contraindications for aspirin or pantoprazole, significant somatic illness, chronic non-steroidal anti-inflammatory drug use, corticosteroid use, pregnancy or change in type or dose of antipsychotic drugs in the previous 2 weeks.

Setting: 10 psychiatric hospitals in The Netherlands; May 2004 to August 2007.

Intervention: Adjuvant aspirin (1000 mg daily) or placebo (adjuvant to antipsychotic medication). All participants received pantoprazole $40 \mathrm{mg}$ daily for gastric protection.

Outcomes: Psychopathology (PANSS total, positive, negative and general psychopathology scores).

Patient follow-up: $83 \%$.

\section{METHODS}

Design: Randomised controlled trial.

Allocation: Unclear.

Blinding: Double blind.

Follow-up period: 3 months.

\section{MAIN RESULTS}

Adjuvant aspirin reduced overall psychopathology compared with placebo in mixed effect models at 3 months (change in mean total PANSS score in last observation carried forward (LOCF) analyses: -9.27 with aspirin vs -5.46 with placebo, difference in unpaired t tests: $+3.81,95 \% \mathrm{CI}-1.10$ to +8.73 ; difference based on linear mixed model: $4.86,95 \%$ CI 0.91 to 8.80; effect size 0.47 ). There was also a reduction in positive symptoms with adjuvant aspirin compared with placebo in mixed effect models (change in mean total PANSS positive subscale score in LOCF analyses: -2.24 with aspirin vs -1.32 with placebo, difference in unpaired t tests: $+0.92,95 \% \mathrm{CI}$ -0.98 to +2.82 ; difference based on linear mixed model: 1.57 , 95\% CI 0.06 to 3.07; effect size 0.39 ). The mixed model did not show differences in negative or general PANSS subscale scores between aspirin and placebo. Aspirin had a greater effect on overall psychopathology in individuals with more altered immune function (ie, lower $\mathrm{T}_{\mathrm{H}} 1 / \mathrm{T}_{\mathrm{H}} 2$ ratios; $\mathrm{p}=0.018$ ). Aspirin significantly reduced overall psychopathology in individuals with the lowest $\mathrm{T}_{\mathrm{H}} 1 / \mathrm{T}_{\mathrm{H}} 2$ ratios (difference in PANSS total score: $7.47,95 \%$ CI 1.97 to 12.98 ) but not in those with the highest $\mathrm{T}_{\mathrm{H}} 1 / \mathrm{T}_{\mathrm{H}} 2$ ratios (difference in PANSS total score: $+2.39,95 \% \mathrm{CI}-3.50$ to +8.28$)$.

\section{CONCLUSIONS}

Adding aspirin to antipsychotic treatment reduces the symptoms of schizophrenia spectrum disorders more than adding placebo.

\section{NOTES}

Randomisation was stratified based on psychiatric centre (referral or non-referral) and relative $\mathrm{T}_{\mathrm{H}} 1 / \mathrm{T}_{\mathrm{H}} 2$ cytokine activity (median interferon $\gamma /$ interleukin 4 ratio).

\section{ABSTRACTED FROM}

Laan W, Grobbee DE, Selten JP, et al. Adjuvant aspirin therapy reduces symptoms of schizophrenia spectrum disorders: results from a randomized, double-blind, placebocontrolled trial. J Clin Psychiatry 2010;71:520-7.

Correspondence to: Wijnand Laan, Julius Center for Health Sciences and Primary Care, University Medical Center Utrecht, PO box 85500, 3508 GA, Utrecht, The Netherlands; w.laan@umculrecht

Source of funding: The Stanley Medical Research Institute.
F levated levels of inflammatory biomarkers have been reported in patients with chronic schizophrenia as well as in patients with first episode psychosis. ${ }^{1}$ These findings support the hypothesised role of the activation of the immune system in the aetiopathogenesis of psychosis. Indeed, cytokines have been shown to influence brain biological pathways relevant to psychosis, including neurotransmitter metabolism, neuroendocrine function and neural plasticity. In this article, Laan and colleagues have investigated the efficacy of adjuvant treatment with acetylsalicylic acid (aspirin), a well-known anti-inflammatory agent, in patients with schizophrenia spectrum disorders. The authors found that adding aspirin to an antipsychotic further reduces psychotic symptoms at 3-month follow-up, when compared with an antipsychotic combined with placebo. It is, however, counterintuitive that the effect of aspirin is more pronounced in patients with the lowest $T_{H} 1 / T_{H} 2$ cytokine balance, that is, in those with less evidence of inflammation before treatment.

Previous studies showing a positive effect of adjuvant anti-inflammatory treatment in patients with schizophrenia have used the selective cyclooxygenase- 2 inhibitor, celecoxib, ${ }^{23}$ whereas this is the first study using acetylsalicylic acid. Both drugs have important adverse effects - cardiovascular and gastrointestinal toxicity, respectively - and therefore the balance between the clinical benefit and adverse effects needs further evaluation.

Overall, these findings support a role of immune activation in the development of psychotic symptoms and the need to develop new treatment strategies targeting inflammation in psychiatric patients. However, more extensive immunological characterisation is required to understand the molecular mechanisms underlying these therapeutic effects.

Valeria Mondelli, Carmine M Pariante

Institute of Psychiatry, Kings College London, London, UK

Competing interests None.

\section{REFERENCES}

1. Potvin S, Stip E, Sepehry AA, et al. Inflammatory cytokine alterations in schizophrenia: a systematic quantitative review. Biol Psychiatry 2008;63:801-8.

2. Akhondzadeh $\mathbf{S}$, Tabatabaee $\mathbf{M}, \mathrm{Amini} \mathrm{H}$, et al. Celecoxib as adjunctive therapy in schizophrenia: a double-blind, randomized and placebo-controlled trial Schizophr Res 2007:90:179-85.

3. Müller N, Krause D, Dehning S, et al. Celecoxib treatment in an early stage of schizophrenia: results of a randomized, double-blind, placebo-controlled trial of celecoxib augmentation of amisulpride treatment. Schizophr Res 2010;121:118-24. 